\title{
Erratum to: Visible Electron Orbits of Carbon Atom
}

\author{
Byungwhan Kim \\ DOI: $10.1007 / \mathrm{s} 13391-012-2165-0$
}

The terminology of "carbon atom" appearing in the title and text must be replaced by the "inner Brillouin zone". The inner Brillouin zone covers both outer regions of carbon atoms and inner substrate. Author apologies for the inconvenience caused to the readers. 